\title{
Gluten sensitivity and epilepsy: a systematic review
}

\author{
Thomas Julian ${ }^{1} \cdot$ Marios Hadjivassiliou² ${ }^{2}$ Panagiotis Zis $^{2}$
}

Received: 9 July 2018 / Revised: 14 August 2018 / Accepted: 17 August 2018 / Published online: 23 August 2018

(c) The Author(s) 2018

\begin{abstract}
Objective The aim of this systematic review was to establish the prevalence of epilepsy in patients with coeliac disease (CD) or gluten sensitivity (GS) and vice versa and to characterise the phenomenology of the epileptic syndromes that these patients present with.

Methodology A systematic computer-based literature search was conducted on the PubMed database. Information regarding prevalence, demographics and epilepsy phenomenology was extracted.

Results Epilepsy is 1.8 times more prevalent in patients with CD, compared to the general population. CD is over 2 times more prevalent in patients with epilepsy compared to the general population. Further studies are necessary to assess the prevalence of GS in epilepsy. The data indicate that the prevalence of CD or GS is higher amongst particular epileptic presentations including in childhood partial epilepsy with occipital paroxysms, in adult patients with fixation off sensitivity (FOS) and in those with temporal lobe epilepsy (TLE) with hippocampal sclerosis. A particularly interesting presentation of epilepsy in the context of gluten-related disorders is a syndrome of coeliac disease, epilepsy and cerebral calcification (CEC syndrome) which is frequently described in the literature. Gluten-free diet (GFD) is effective in the management of epilepsy in 53\% of cases, either reducing seizure frequency, enabling reduced doses of antiepileptic drugs or even stopping antiepileptic drugs. Conclusion Patients with epilepsy of unknown aetiology should be investigated for serological markers of gluten sensitivity as such patients may benefit from a GFD.
\end{abstract}

Keywords CEC $\cdot \mathrm{CD} \cdot$ Coeliac $\cdot$ Celiac $\cdot$ Epilepsy $\cdot$ Gluten

\section{Introduction}

Gluten-related disorders (GRDs) represent a spectrum of diverse clinical manifestations triggered by the ingestion of gluten. Although the gastrointestinal manifestations of gluten sensitivity are the most well studied and popularly recognised, there are a range of debilitating neurological manifestations of gluten sensitivity which are increasingly established as the cause of significant disability.

Coeliac disease (CD) is the best recognised amongst these disorders, affecting around $1 \%$ of the population [1]. CD describes patients with primarily small bowel enteropathy in genetically susceptible individuals after exposure to the

Thomas Julian

thjulian07@gmail.com

1 Sheffield Institute for Translational Neuroscience, University of Sheffield, 385a Glossop Rd, Sheffield S10 2HQ, UK

2 Academic Department of Neurosciences, Sheffield Teaching Hospitals NHS Trust, Sheffield, UK protein gliadin [2]. Non-coeliac gluten sensitivity (NCGS) refers to patients with primarily gastrointestinal symptoms related to the ingestion of wheat, barley and rye who do not have enteropathy but who symptomatically benefit from a gluten-free diet (GFD) [3]. Amongst patients presenting to neurology, gastrointestinal symptoms are comparatively rare and thus this definition is unhelpful [4]. Therefore, for neurological purposes we refer to patients as being gluten sensitive (GS), which is defined by positive serology in the form of anti- gliadin IgG and IgA (AGA), transglutaminase (tTG) or endomysial antibodies (EMA) and the presence of a range of extra-intestinal symptoms, but without an abnormal bowel biopsy which is diagnostic of coeliac disease.

Gluten sensitivity is associated with a number of neurological conditions including ataxia [5], headaches with white matter abnormalities on MR imaging [6], peripheral neuropathy [7] and epilepsy [8]. Perhaps the first description of an association between gluten sensitivity and epilepsy was in an article published in 1956 which described a case series of 'fits' in children who the author described to be 
afflicted with behavioural and gastrointestinal disturbance linked to ingestion of gluten but without diagnostic testing [9]. Epileptic seizures presenting in the context of gluten sensitivity encompass the full spectrum of epilepsy and include both patients with and without overt brain pathology who may or may not respond to antiepileptic drugs (AED). This spectrum includes a range of interesting pathological features including a well-defined syndrome of $\mathrm{CD}$, epilepsy and cerebral calcifications (CEC) [10]; hippocampal sclerosis and temporal lobe epilepsy (TLE) in the context of gluten sensitivity [11]; and those who apparently display no pathological clues to the specific cause of the epilepsy.

The aim of this study was to systematically review the current literature in order to establish the prevalence of epilepsy in patients with $C D$ and GS, the prevalence of $C D$ and GS in patients with epilepsy and characterise the phenomenology of the epileptic syndromes that these patients present with.

\section{Methodology}

\section{Protocol}

This review is not registered on a public database. It is registered on the database of dissertation projects for the MSc in Clinical Neurology at the University of Sheffield.

\section{Search strategy}

A systematic PubMed search was performed on the 14th of December 2017. For the search, two medical subject headings (MeSH terms) were used. Term A was "coeliac" or "celiac" or "gluten". Term B was "epilepsy" or "epileptic" or "epilepsia" or "epilepticus" or "myoclonus" or "myoclonic". No restrictions were applied in our search strategy.

\section{Inclusion and exclusion criteria}

Articles eligible to be included in the review were required to meet the following criteria:

1. The study subjects were diagnosed with epilepsy of unknown aetiology and gluten sensitivity.

2. The study subjects were human.

3. The study contained original data.

4. The study was available as a full text, English language article or contained utilisable information in an English language abstract.

The following were excluded:
1. Articles which remained unavailable despite attempts to contact the author and requests for access made to the British Library.

2. Studies detailing epilepsy which is secondary to another neurological insult.

3. Articles detailing gluten sensitivity which was not confirmed using positive serology as a minimum standard.

4. Myoclonus which was reference to anything other than a seizure type in the context of epilepsy.

All studies were screened and assessed for eligibility by two authors independently. Details of the inclusion process are detailed in Fig. 1.

\section{Data collection process}

Data were extracted from each study in a structured coding scheme using Google Sheets and included: location of study; type of study; population size, age and sex; the nature of gluten sensitivity; the classification of epilepsy; the age of onset of epilepsy and gluten sensitivity; imaging results; serological results; brain and bowel biopsy results; blood test results and epilepsy response to treatment including glutenfree diet, antiepileptic medication and surgery.

For the purposes of this review, gluten sensitivity was considered to be $\mathrm{CD}$ if it was categorised so by satisfying the Modified Marsh Criteria (Marsh III) [12]. Gluten sensitivity was defined as presence of one or more of the following: Positive serum AGA (IgG and/or IgA), anti-tTG or antiEMA, without Marsh III on bowel biopsy or in the absence of biopsy. Consequently, some patients classified with gluten sensitivity who had not undergone a bowel biopsy in this review may have unidentified CD. Epilepsy of unknown aetiology in patients without a comorbid condition which may result in seizures (e.g. head injury, brain surgery, brain tumour etc) was included.

\section{Synthesis of results}

Frequencies and descriptive characteristics extracted were calculated using Google Sheets. These were calculated as means and $95 \%$ confidence intervals (CI). This study is reported in accordance with the Preferred Reporting Items for Systematic Reviews and Meta-Analysis (PRISMA) guidelines.

\section{Assessment of bias}

None of the studies included in this review are randomised control trials or interventional studies for which risk of bias tools are available. A risk of bias tool was therefore not used. 
Fig. 1 A PRISMA chart detailing the inclusion process

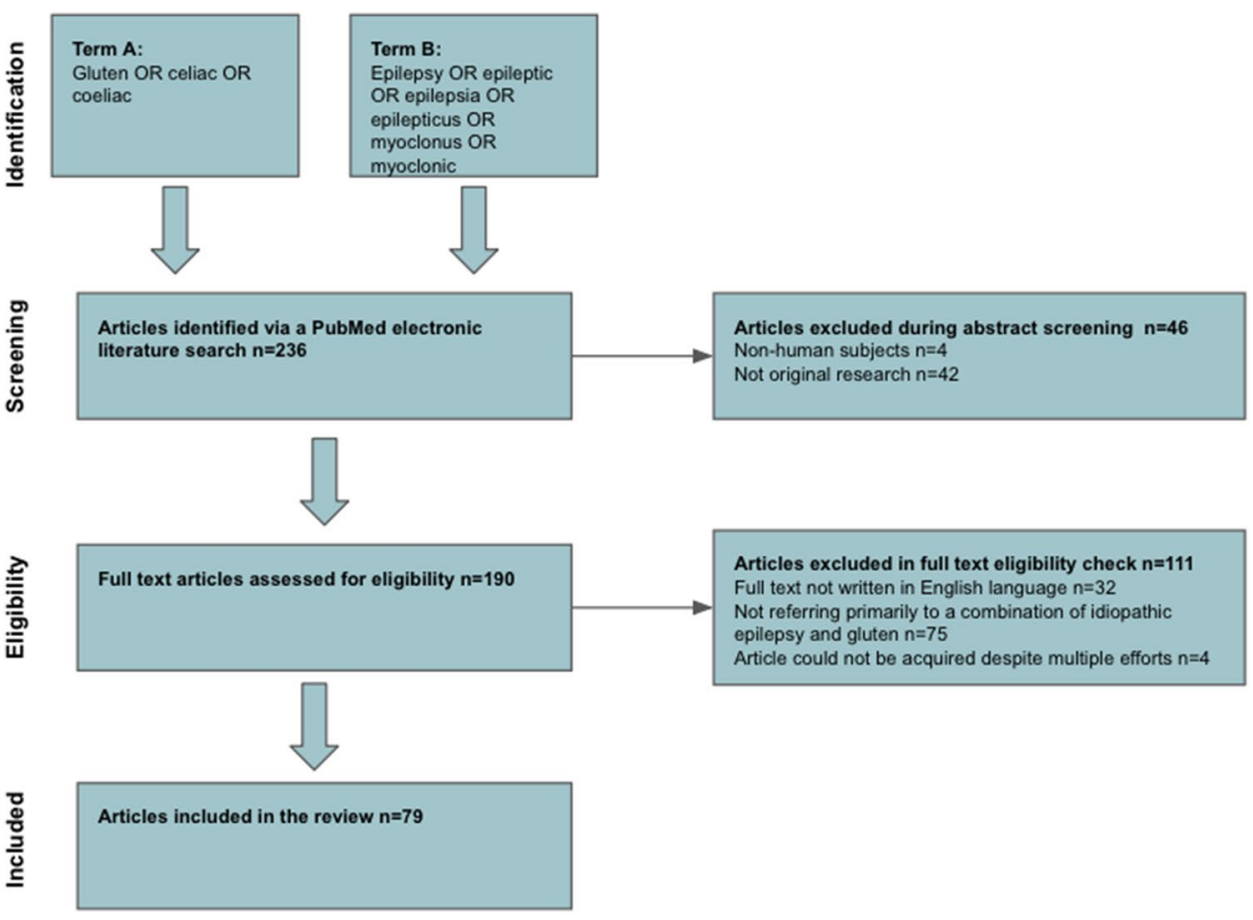

\section{Compliance with ethical guidelines}

This article is based upon previously published studies. The article is in compliance with the journal's ethical guidelines.

\section{Results}

\section{Selected studies}

The search strategy identified 236 articles. A total of 46 articles were excluded during the title and abstract screening stage. A total of 111 articles were excluded during the eligibility assessment. Thus, 79 articles published between 1970 and 2017 qualified for inclusion in this review studying a total of 39,579 individuals with either epilepsy, coeliac disease or both $[8,10,11,13-88]$. Of these, 26 were case reports, 7 were case-control studies and the remaining 46 articles were prospective or retrospective case series (most of which were consecutively recruited cohort studies). Whilst the studies are published in a range of locations worldwide, a disproportionate number come from Italy $(n=24)$ and Turkey $(n=8)$. Figure 1 illustrates the study selection process.

\section{Epidemiology of gluten sensitivity and CD associated epilepsy}

Thirteen studies detailed the prevalence of CD amongst patients with epilepsy of unknown aetiology [16, 21, 33, $37,43,45,48,51,59,63,67,69,74]$. The total pooled prevalence was $2.1 \%$ (95\% CI $1.64-2.64 \%, n=3389$ ). Amongst exclusively paediatric populations the prevalence was $1.83 \%$ (95\% CI 1.26-2.56\%, $n=1804$ ) whilst in exclusively adult populations the prevalence was $2.27 \%$ (95\% CI $1.52-3.24 \%, n=1280$ ). Seven studies detailed prevalence of serologically confirmed gluten sensitivity amongst patients with epilepsy of unknown aetiology, and the pooled prevalence was 3.8\% (95\% CI 2.65-5.34\%, $n=861)[28,37,41,45,48,63,68]$.

Fifteen studies detailed prevalence of epilepsy of unknown aetiology amongst CD sufferers [8, 13, 19, 20, $25,38,42,54,56,57,61,65,74,86,88]$. The pooled prevalence was $1.14 \%$ (95\% CI $1.03-1.26 \%, n=33,217)$. Two studies detailed prevalence of epilepsy amongst serologically confirmed gluten sensitivity. The pooled prevalence was $0.93 \%$ (95\% CI $0.43-1.77 \%, n=963)$. Both of these figures are raised relative to a general population point prevalence of $0.64 \%$ [89].

These figures illustrate that there is an increased prevalence of $\mathrm{CD}$ amongst those with epilepsy and viceversa. They also demonstrate and increased prevalence of epilepsy amongst those with GS. A large, populationbased study conducted in Sweden by Ludvigsson et al. $(n=28,885)$ demonstrated a bidirectional relationship 
between development of CD and epilepsy which illustrates that each population is at increased risk of developing the other condition [8].

\section{Epilepsy classification}

The classification of the epilepsy presentations was reported in 45 of the articles $[8,10,11,13,15,16,19-22,25-30,32$, $33,36,38,41-44,48,49,51,54,56,57,60-69,74,75,77$, $81,86-88,, 60-69,74,75,77,81,86-88]$. Of these, 25 could be used to calculate the prevalence of focal epilepsy amongst patients with epilepsy $[8,13,15,16,18-21,28,33,36,41$, $43,45,48,51,54,56,57,61,63,65,74,87,88]$. Amongst 351 patients, $59 \%$ presented with focal seizures. Based on this, it appears that there is a slightly greater tendency for epilepsy in the context of CD/GS to be focal [90].

\section{Presentation}

Forty-one studies detailed the age of onset of epilepsy in those with CD/GS [11, 14-18, 22, 34-36, 39, 40, 45, 47, 52-56, 58, 60-64, 66, 71, 73, 76-80, 82, 83, 87, 70, 71, 73, $76-80,82,83,87]$. This gave a pooled mean age of onset of $12(n=161)$. Eleven studies $(n=52)$ provided information which enabled calculation of the total number of patients suffering CD and epilepsy who suffered gastrointestinal symptoms [16, 21, 33, 37, 43, 45, 48, 51, 59, 63, 64, 67, $69,74]$. This gave a pooled figure of $40 \%$ of patients having no gastrointestinal symptoms and who, therefore, only presented with neurological symptoms resulting in the diagnosis of $\mathrm{CD}$. This is comparable to other neurological manifestations of gluten sensitivity in which gastrointestinal symptoms are rarely present [4]. Nevalainen et al. investigated the risk of death in patients suffering epilepsy who also had serum markers indicative of $\mathrm{CD} /$ gluten sensitivity and found that there was no increased risk of death in this patient cohort relative to those who have epilepsy but no gluten sensitivity [23].

\section{Management}

Thirty-two papers discuss the therapeutic impact of glutenfree diet $[10,13,15-18,22,31,34,36,39,45-47,50,53$, $55,59-61,66,67,71,73,77,80,81,83-85]$. For the purpose of this review, a positive response was defined as either decreased frequency of seizures with GFD, cessation of seizures with GFD, successful reduction of AED with initiation of GFD or cessation of AED following the introduction of GFD. Amongst studies which reported response to GFD in a consecutively recruited series, $53 \%$ of patients were responsive to a GFD and the remaining 47\% deemed unresponsive $(n=34)$ [13, 15, 16, 36, 49, 61, 67]. The response to GFD could reflect resolution/reduction of a neurological insult caused by gluten ingestion or be the result of improved absorption of AED due to resolution of gastrointestinal disturbance [69]. Data regarding response to AED alone is only available for the CEC syndrome subset. These data indicate that GFD can be an effective management of seizures in patients with gluten sensitivity.

\section{CEC syndrome}

\section{Presentation}

Of the 79 studies included in this review, 30 detailed cases of CEC syndrome making it the best classified presentation of epilepsy in the context of CD and gluten sensitivity [10, 14, $17,32,34,36,44,50,52,55,60,62,64,66,70-85]$. Sixteen of these papers are case reports and 14 are retrospective or prospective case series. These patients generally have focal, medically refractory epilepsy and show parieto-occipitally brain calcifications on CT or MRI. In $82 \%$ of cases, cerebral calcifications were located posteriorly $(n=131)$. In the remainder, calcifications were either frontal, temporal or in a very small minority in sub-cortical areas. The classification of epilepsy was reported in 28 articles, $78 \%$ of seizures in CEC syndrome reported in the available literature are focal in nature $(n=121)[14,17,32,34,36,50,52,55,60,62,64$, $66,70-85]$. Fourteen studies detailed further localisation of the focal epilepsy and $71 \%$ of this was occipital in nature $(n=85)[14,17,36,50,52,55,60,64,71,74,76,81,83$, 85]. Our search identified just one study that investigated a cohort of GFD treated, mostly paediatric CD patients for brain lesions independent of neurological signs [65]. The authors found no calcifications on CT, but MRI showed bilateral or unilateral T2-hyperintensive white-matter lesions in $20 \%$ of patients $(n=75)$. These lesions were independent of specific neurological presentations. This study does not support an increased prevalence of cerebral calcifications in $\mathrm{CD}$ patients but does demonstrate frequent brain imaging abnormalities in CD. The failure to identify calcifications is not surprising considering the rarity of CEC syndrome.

\section{Pathology}

Several studies show that despite improvements in seizure control with GFD there is no reduction in calcification size $[10,71,73,80]$. This has led some researchers to suggest that the calcifications may represent an epiphenomenon rather than being causative. One speculated that the cause of the development of calcifications is folate deficiency. Primarily, this link has been made due to the co-occurrence of folate deficiency and cerebral calcifications in other situations, for instance during methotrexate therapy or in primary cerebral folate deficiency [34]. Calvani et al., have previously demonstrated decreased folic acid transport across the 
blood brain barrier as well as decreased intestinal absorption of folic acid in case report and speculate this to be part of the pathological process [66]. Whilst there are indeed numerous examples of co-occurring folate deficiency and CEC syndrome $[10,34,44,52,71,73,74,76-82]$, it is not a universal finding and there are numerous examples of patients with CEC syndrome and folate values within the normal range $[44,64,79,81]$. Therefore, the relationship between folate and calcifications is inconsistent.

A case report by Johnson et al., identified high levels of IgA directed by transglutaminase isoenzyme 6 (TG6) in the patient's serum [34]. Raised levels of TG6 have previously been shown to be elevated in other neurological manifestations of gluten sensitivity and represents a potential marker of neurological involvement [91]. This study also used indirect immunofluorescence on monkey brain which identified cerebral and cerebellar binding of $\operatorname{IgA}$ which demonstrates potential autoimmunity to the parenchyma of the brain. Brain biopsy was conducted in CEC syndrome patients in two case reports. One case reported a biopsy following right anterior temporal lobectomy with pathology confirming hippocampal sclerosis with severe cell loss and gliosis in CA1/CA3/CA4 [17]. The second case report by Bye et al., demonstrated cortical vascular abnormality with patchy pial angiomatosis, fibrosed veins and large, jagged microcalcification [76].

\section{Management}

The more descriptive nature of the CEC syndrome studies enables insight into the patient's response to treatment. Response to AED alone appears to be poor amongst patients with CEC syndrome, with $73 \%$ unresponsive to treatment ( $n=74)$ [10, 32, 36, 44, 60, 64, 74, 75, 77, 81]. Response to GFD appears to be more effective, with $53 \%$ of patients managed with GFD demonstrating a good response $(n=47)$ $[10,36,60,77,81]$. Interestingly, three prospective cohort studies appear to have demonstrated an inverse relationship between effectiveness of GFD and duration of epilepsy prior to GFD, perhaps due to increasing neurological damage [36, $60,81]$.

There are four reported cases of surgical resection of cerebral calcifications with the aim to resolve intractable seizures $[17,36,52,77]$. Of these, one was resistant to surgery, two were responsive to surgery and one responded to a combination of surgical resection and GFD. Whilst two patients did respond to surgery, one continued to suffer seizures but of lesser severity, and one was commenced gluten-free diet immediately following surgery. Consequently, it is feasible that the improvement is owed to GFD rather than to surgical resection. Based on the very limited data available, surgical resection of calcifications alone does not resolve epilepsy in CEC syndrome. These findings must be interpreted with caution as the data include studies using selective populations (non-consecutive case series and case reports).

\section{Other specific epilepsy syndromes in GS and CD}

A small number of papers have investigated specific epilepsy syndromes in relation to GS and CD. These studies are limited by their small population sizes but demonstrate a possible interesting link between GS/CD and some specific syndromes.

\section{Childhood partial epilepsy with occipital paroxysms}

Three articles investigated the prevalence of CD amongst those with childhood partial epilepsy with occipital paroxysms [21, 26, 64]. According to these, the pooled prevalence of CD amongst such individuals is $10.41 \%$ (95\% CI $5.11-18.32 \%, n=96)$. This is considerably higher than the prevalence $\mathrm{CD}$ amongst all epilepsy of unknown aetiology.

\section{Children with occipital lobe epilepsy}

Dai et al. conducted a case-control study with a cohort of children with both generalised and focal epilepsy identified to be occipital in origin on EEG [27]. This study discovered $2.22 \%(n=90)$ of the patient cohort also had CD which was significantly higher than the healthy control group. The two patients with $\mathrm{CD}$ were also monotherapy resistant.

\section{Adult patients with fixation off sensitivity (FOS)}

Fattouch et al. investigated adult epileptic patients with FOS [32]. These patients all presented with simple and complex focal seizures, which were posterior in onset according to EEG. This study showed a prevalence of CD of $20 \%(n=15)$ amongst this patient group.

\section{Progressive myoclonic epilepsy (PME)}

Franceschetti et al., investigated comorbid conditions amongst patients with PME of undetermined cause by collecting existing medical record data [29]. This study identified that $0.98 \%(n=204)$ of the patient cohort suffered comorbid CD. This data is limited because as previously established most patients are not symptomatic for $\mathrm{CD}$, and so it is likely checking medical records alone is not sufficient to rule out $\mathrm{CD}$ and GS as the condition will likely be undiagnosed.

\section{Temporal lobe epilepsy (TLE) with hippocampal sclerosis}

Peltola et al. studied the relationship between temporal lobe epilepsy (TLE) with hippocampal sclerosis, and gluten 
sensitivity [11]. Autoimmunity has previously been implicated in the pathophysiology of hippocampal sclerosis and thus this study investigated GS antibodies in a prospective study of patients with refractory TLE with or without hippocampal sclerosis as well as in those with extra-temporal epilepsy. This study identified a significantly raised prevalence of gluten sensitivity seropositivity in those with TLE and hippocampal sclerosis with a figure of $43.75 \%(n=16)$. These patients were then tested for CD, with three patients displaying histology for CD (18.75\%) and four showing early changes associated with CD. This is the only study linking gluten sensitivity or CD to hippocampal sclerosis and TLE. This study is limited by its small population. As previously mentioned, hippocampal sclerosis was also present in the biopsy of a patient suffering CEC syndrome in a separate study [17].

\section{Summary}

In tandem with studies regarding CEC syndrome, these data appear to support a hypothesis of a selective vulnerability of specific brain regions to damage in the context of gluten sensitivity or coeliac disease.

\section{Assessment of bias}

None of the studies included in this review are randomised control trials or interventional studies for which risk of bias tools are available. All articles were reviewed by two authors independently and their judgements regarding inclusion matched in all cases. Case reports are low level evidence with a high risk of bias. The use of this data is summarised in the "Limitations" section of this review.

\section{Conclusions}

This systematic review has identified the following key points:

1. There is an increased prevalence of CD amongst patients with epilepsy and an increased prevalence of epilepsy amongst those with $\mathrm{CD}$ or gluten sensitivity.

2. Patients with CD presenting with neurological symptoms often suffer no gastrointestinal symptoms.

3. There appears to be a stronger link between some epileptic presentations and GS or CD than others. Future studies should not treat epilepsy as though it is homogenous when investigating its relationship with GS or CD.

4. Gluten-free diet is an effective management of epilepsy in those with epilepsy due to GS/CD.

5. CEC syndrome is the best characterised epileptic presentation linked to $\mathrm{CD}$.
6. Patients with epilepsy of unknown aetiology should have their serum screened for AGA, anti tTG and EMA. This is especially important in patients suffering with AED resistant occipital lobe seizures. It is likely that there are many patients who are being treated with AED polytherapy who can be managed with a GFD alone or with GFD and reduced AED.

7. There is a need to study the prevalence of TG6 antibodies in patients with epilepsy to identify whether anti-TG6 could be used to identify individuals at risk of epilepsy due to their gluten sensitivity.

The results of this review are highly relevant to Dieticians and Gastroenterologists as well as Neurologists. It is important that epilepsy is more broadly recognised within the spectrum of gluten-related disorders as these patients can be managed effectively if identified. However, clinicians must approach these cases with caution so as to not incorrectly diagnose epilepsy in those with gluten intolerance who have epilepsy mimics such as syncope, psychogenic non-epileptic seizures or migraine, amongst others. Clinicians must also recognise the limitations to the specificity of the GS/CD serum markers and take into account the full clinical picture when proceeding with diagnosis.

\section{Limitations}

- There is a significant deal of low quality evidence utilised in this review due to the large number of case reports. Studies which use selected patient groups were utilised to evaluate: Response to treatment, descriptive peculiarities where it is explicitly stated that a case report was used and for results regarding CEC syndrome. All other results were calculated using studies with consecutively recruited patient cohorts or entire populations only.

- Not all studies biopsied their GS patients. The patients may therefore have suffered CD. This may cause underestimation of the link between CD and epilepsy.

- Some studies reviewed medical records rather than prospectively investigating for $\mathrm{CD} / \mathrm{GS}$. Often patients do not suffer classical symptoms of GS/CD and therefore will not be diagnosed. Therefore, the prevalence of CD/GS amongst epilepsy may be an underestimate.

- This study addressed the relationship between CD/ GS and epilepsy of unknown aetiology. There were no restrictions placed upon date of publication of articles included in this review and as such some studies are dated as far back as 1970. As the understanding of the causes of epilepsy has developed it is possible that some patients included within the studies forming this review would now be recognised to have a clear aetiology and therefore will be incorrectly classified. 
- Four articles were excluded because they could not be retrieved.

- Articles for inclusion in this review were retrieved via a search in a single electronic database.

Acknowledgements We are sincerely thankful to Dr Stamatina Iliodromiti for her contribution in the statistical analysis of the data. Dr Zis is sincerely thankful to the Ryder Briggs Fund.

This is a summary of independent research carried out at the NIHR Sheffield Biomedical Research Centre (Translational Neuroscience). The views expressed are those of the authors and not necessarily those of the NHS, the NIHR or the Department of Health.

\section{Compliance with ethical standards}

Conflicts of interest None of the authors have any competing interests.

Open Access This article is distributed under the terms of the Creative Commons Attribution 4.0 International License (http://creativeco mmons.org/licenses/by/4.0/), which permits unrestricted use, distribution, and reproduction in any medium, provided you give appropriate credit to the original author(s) and the source, provide a link to the Creative Commons license, and indicate if changes were made.

\section{References}

1. Mustalahti K, Catassi C, Reunanen A et al (2010) The prevalence of celiac disease in Europe: results of a centralized, international mass screening project. Ann Med 42:587-595

2. Fasano A, Catassi C (2001) Current approaches to diagnosis and treatment of celiac disease: an evolving spectrum. Gastroenterology 120:636-651

3. Sapone A, Bai JC, Ciacci C et al (2012) Spectrum of glutenrelated disorders: consensus on new nomenclature and classification. BMC Med 10:13

4. Hadjivassiliou M, Sanders DS, Grünewald RA et al (2010) Gluten sensitivity: from gut to brain. Lancet Neurol 9:318-330

5. Hadjivassiliou M (2012) Immune mediated acquired ataxias. Handb Clin Neurol 103:189-199

6. Gabrielli M, Cremonini F, Fiore G et al (2003) Association between migraine and Celiac disease: results from a preliminary case-control and therapeutic study. Am J Gastroenterol 98:625-629

7. Zis P, Rao DG, Sarrigiannis PG et al (2017) Transglutaminase 6 antibodies in gluten neuropathy. Dig Liver Dis 49:1196-1200

8. Ludvigsson JF, Zingone F, Tomson T et al (2012) Increased risk of epilepsy in biopsy-verified celiac disease: a population-based cohort study. Neurology 78:1401-1407

9. Guy Daynes (1956) Bread and tears—-naughtiness, depression and fits due to wheat sensitivity. Proc R Soc Med 49:391-394

10. Gobbi G, Ambrosetto P, Zaniboni MG et al (1992) Celiac disease, posterior cerebral calcifications and epilepsy. Brain Dev 14:23-29

11. Peltola M, Kaukinen K, Dastidar P et al (2009) Hippocampal sclerosis in refractory temporal lobe epilepsy is associated with gluten sensitivity. J Neurol Neurosurg Psychiatry 80:626-630

12. Marsh N, Johnson MW, Rostami M (2015) K. Mucosal histopathology in celiac disease: a rebuttal of Oberhuber's sub-division of Marsh III. Gastroenterol Hepatol Bed Bench 8:99-109

13. Chapman RW, Laidlow JM, Colin-Jones D et al (1978) Increased prevalence of epilepsy in coeliac disease. Br Med J 2:250-251
14. Bonanni P, Negrin S, Antoniazzi L et al (2017) Clinical implications of interictal epileptiform discharges in cognitive functioning in CEC syndrome with evolution into epileptic encephalopathy. Neurocase 23:230-238

15. Sel ÇG, Aksoy E, Aksoy A et al (2017) Neurological manifestations of atypical celiac disease in childhood. Acta Neurol Belg 117:719-727

16. Bashiri H, Afshari D, Babaei N et al (2016) Celiac Disease and epilepsy: the effect of gluten-free diet on seizure control. Adv Clin Exp Med 25:751-754

17. Struck AF, Beinlich BR, Rutecki PA (2015) A case of celiac disease, epilepsy, and cerebral calcifications with temporal lobe epilepsy. WMJ 114:116-117

18. Işikay S, Hizli Ş, Kocamaz H (2015) Celiac disease and juvenile absence epilepsy. Pediatr Emerg Care 31:e19-e20

19. Işikay S, Kocamaz H (2015) The neurological face of celiac disease. Arq Gastroenterol 52:167-170

20. Işıkay S, Kocamaz H, Sezer S et al (2015) The frequency of epileptiform discharges in celiac disease. Pediatr Neurol 53:78-82

21. Işikay S, Hizli Ş, Yilmaz K (2014) Prevalence of celiac disease in Turkish children with idiopathic epilepsy. Iran J Pediatr 24:280-284

22. Casciato S, Morano A, Albini M et al (2015) Cryptogenic focal epilepsy and "hidden" celiac disease in adulthood: a causal or accidental link? Int J Neurosci 125:913-917

23. Nevalainen O, Auvinen A, Ansakorpi H et al (2014) Autoimmunity-related immunological serum markers and survival in a tertiary care cohort of adult patients with epilepsy. Epilepsy Res 108:1675-1679

24. Soós Z, Salamon M, Erdei K et al (2014) LADA type diabetes, celiac diasease, cerebellar ataxia and stiff person syndrome. A rare association of autoimmune disorders. Ideggyogy Sz 67:205-209

25. Ong MS, Kohane IS, Cai T,, et al (2014) Population-level evidence for an autoimmune etiology of epilepsy. JAMA Neurol 71:569-574

26. Iş1kay S, Kocamaz H (2014) Prevalence of celiac disease in children with idiopathic epilepsy in southeast Turkey. Pediatr Neurol 50:479-481

27. Dai AI, Akcali A, Varan C et al (2014) Prevalence of resistant occipital lobe epilepsy associated with celiac disease in children. Childs Nerv Syst 30:1091-1098

28. Vieira C, Jatobá I, Matos M et al (2013) Prevalence of celiac disease in children with epilepsy. Arq Gastroenterol 50:290-296

29. Franceschetti S, Michelucci R, Canafoglia L et al (2014) Progressive myoclonic epilepsies: definitive and still undetermined causes. Neurology 82:405-411

30. Sarrigiannis PG, Hoggard N, Aeschlimann D et al (2014) Myoclonus ataxia and refractory coeliac disease. Cerebellum Ataxias 1:11

31. Vitelli O, Miano S, Tabarrini A et al (2014) Epilepsy and sleepdisordered breathing as false friends: a case report. J Child Neurol 29:114-117

32. Fattouch J, Casciato S, Lapenta L et al (2013) The spectrum of epileptic syndromes with fixation off sensitivity persisting in adult life. Epilepsia 7:59-65

33. Djurić Z, Nagorni A, Jocić-Jakubi B et al (2012) Celiac disease prevalence in epileptic children from Serbia. Turk J Pediatr $54: 247-250$

34. Johnson AM, Dale RC, Wienholt L et al (2013) Coeliac disease, epilepsy, and cerebral calcifications: association with TG6 autoantibodies. Dev Med Child Neurol 55:90-93

35. Javed S, Safdar A, Forster A et al (2012) Refractory coeliac disease associated with late onset epilepsy, ataxia, tremor and progressive myoclonus with giant cortical evoked potentials-a case report and review of literature. Seizure 21:482-485 
36. Licchetta L, Bisulli F, Di Vito L et al (2011) Epilepsy in coeliac disease: not just a matter of calcifications. Neurol Sci 32:1069-1074

37. Ertekin V, Selimoğlu MA, Tan H et al (2010) Prevalence of celiac disease in a sample of Turkish children with epilepsy. Pediatr Neurol 42:380-381 (380; author reply)

38. Bürk K, Farecki ML, Lamprecht G et al (2009) Neurological symptoms in patients with biopsy proven celiac disease. Mov Disord 24:2358-2362

39. Sallem FS, Castro LM, Jorge C et al (2009) Gluten sensitivity presenting as myoclonic epilepsy with cerebellar syndrome. Mov Disord 24:2162-2163

40. Ryan AM, Ryan J, Wan-Ahmed M et al. (2009) Vacuolar leucoencephalopathy and pulvinar sign in association with coeliac disease. BMJ Case Rep

41. Emami MH, Taheri H, Kohestani S et al (2008) How frequent is celiac disease among epileptic patients? J Gastrointestin Liver Dis 17:379-382

42. Briani C, Zara G, Alaedini A et al (2008) Neurological complications of celiac disease and autoimmune mechanisms: a prospective study. J Neuroimmunol 195:171-175

43. Ruggieri M, Incorpora G, Polizzi A et al (2008) Low prevalence of neurologic and psychiatric manifestations in children with gluten sensitivity. J Pediatr 152:244-249

44. Della Nave R, Magaudda A, Michelucci R et al (2007) Wholebrain histogram and voxel-based analyses of apparent diffusion coefficient and magnetization transfer ratio in celiac disease, epilepsy, and cerebral calcifications syndrome. AJNR 28:479-485

45. Mavroudi A, Xinias I, Papastavrou T et al (2007) Increased prevalence of silent celiac disease among Greek epileptic children. Pediatr Neurol 36:165-169

46. Harper E, Moses H, Lagrange A (2007) Occult celiac disease presenting as epilepsy and MRI changes that responded to gluten-free diet. Neurology 68:533-534

47. Canales P, Mery VP, Larrondo FJ et al (2006) Epilepsy and celiac disease: favorable outcome with a gluten-free diet in a patient refractory to antiepileptic drugs. Neurologist 12:318-321

48. Dalgiç B, Dursun I, Serdaroğlu A et al (2006) Latent and potential celiac disease in epileptic Turkish children. J Child Neurol 21:6-7

49. Mavroudi A, Karatza E, Papastavrou T et al (2005) Successful treatment of epilepsy and celiac disease with a gluten-free diet. Pediatr Neurol 33:292-295

50. Díaz RM, González-Rabelino G, Delfino A (2005) Epilepsy, cerebral calcifications and coeliac disease. The importance of an early diagnosis. Rev Neurol 40:417-420

51. Ranua J, Luoma K, Auvinen A et al (2005) Celiac disease-related antibodies in an epilepsy cohort and matched reference population. Epilepsy Behav 6:388-392

52. Nakken KO, Røste GK, Hauglie-Hanssen E (2005) Coeliac disease, unilateral occipital calcifications, and drug-resistant epilepsy: successful lesionectomy. Acta Neurol Scand 111:202-204

53. Siqueira Neto JI, Costa AC, Magalhães FG et al (2004) Neurological manifestations of celiac disease. Arq Neuropsiquiatr 62:969-972

54. Vaknin A, Eliakim R, Ackerman Z et al (2004) Neurological abnormalities associated with celiac disease. J Neurol 251:1393-1397

55. Pfaender M, D'Souza WJ, Trost N et al (2004) Visual disturbances representing occipital lobe epilepsy in patients with cerebral calcifications and coeliac disease: a case series. J Neurol Neurosurg Psychiatry 75:1623-1625

56. Pengiran Tengah DS, Holmes GK, Wills AJ (2004) The prevalence of epilepsy in patients with celiac disease. Epilepsia 45:1291-1293
57. Zelnik N, Pacht A, Obeid R et al (2004) Range of neurologic disorders in patients with celiac disease. Pediatrics 113:1672-1676

58. Pratesi R, Modelli IC, Martins RC et al (2003) Celiac disease and epilepsy: favorable outcome in a child with difficult to control seizures. Acta Neurol Scand 108:290-293

59. Essid M, Trabelsi K, Jerbi E et al (2003) Villous atrophy and idiopathic epilepsy. Tunis Med 81:270-272

60. Arroyo HA, De Rosa S, Ruggieri V et al (2002) Epilepsy, occipital calcifications, and oligosymptomatic celiac disease in childhood. J Child Neurol 17:800-806

61. Volta U, De Giorgio R, Petrolini N et al (2002) Clinical findings and anti-neuronal antibodies in coeliac disease with neurological disorders. Scand J Gastroenterol 37:1276-1281

62. Santos CH, Almeida IL, Gomes MD et al (2002) Bilateral occipital calcification, epilepsy and coeliac disease: case report. Arq Neuropsiquiatr 60:840-843

63. Luostarinen L, Dastidar P, Collin P et al (2001) Association between coeliac disease, epilepsy and brain atrophy. Eur Neurol 46:187-191

64. Labate A, Gambardella A, Messina D et al (2001) Silent celiac disease in patients with childhood localization-related epilepsies. Epilepsia 42:1153-1155

65. Kieslich M, Errázuriz G, Posselt HG et al (2001) Brain whitematter lesions in celiac disease: a prospective study of 75 diettreated patients. Pediatrics 108:E21

66. Calvani M Jr, Parisi P, Guaitolini C et al (2001) Latent coeliac disease in a child with epilepsy, cerebral calcifications, drug-induced systemic lupus erythematosus and intestinal folic acid malabsorption associated with impairment of folic acid transport across the blood-brain barrier. Eur J Pediatr 160:288-292

67. Salur L, Uibo O, Talvik I et al (2000) The high frequency of coeliac disease among children with neurological disorders. Eur J Neurol 7:707-711

68. Lahat E, Broide E, Leshem M et al (2000) Prevalence of celiac antibodies in children with neurologic disorders. Pediatr Neurol 22:393-396

69. Cronin CC, Jackson LM, Feighery C et al (1998) Coeliac disease and epilepsy. QJM 91:303-308

70. Bernasconi A, Bernasconi N, Andermann F et al (1998) Celiac disease, bilateral occipital calcifications and intractable epilepsy: mechanisms of seizure origin. Epilepsia 39:300-306

71. Hernández MA, Colina G, Ortigosa L (1998) Epilepsy, cerebral calcifications and clinical or subclinical coeliac disease. Course and follow up with gluten-free diet. Seizure 7:49-54

72. Baquero M, Narciso ML, García M et al (1995) Celiac disease with occipital calcifications: 2 late cases. Med Clin (Barc) 105:781-783

73. Lea ME, Harbord M, Sage MR (1995) Bilateral occipital calcification associated with celiac disease, folate deficiency, and epilepsy. AJNR Am J Neuroradiol 16:1498-1500

74. Fois A, Vascotto M, Di Bartolo RM et al (1994) Celiac disease and epilepsy in pediatric patients. Childs Nerv Syst 10:450-454

75. Bardella MT, Molteni N, Prampolini L et al (1994) Need for follow up in coeliac disease. Arch Dis Child 70:211-213

76. Bye AM, Andermann F, Robitaille Y et al (1993) Cortical vascular abnormalities in the syndrome of celiac disease, epilepsy, bilateral occipital calcifications, and folate deficiency. Ann Neurol 34:399-403

77. Magaudda A, Dalla Bernardina B, De Marco P et al (1993) Bilateral occipital calcification, epilepsy and coeliac disease: clinical and neuroimaging features of a new syndrome. J Neurol Neurosurg Psychiatry 56:885-889

78. Piattella L, Zamponi N, Cardinali C et al (1993) Endocranial calcifications, infantile celiac disease, and epilepsy. Childs Nerv Syst 9:172-175 
79. Tiacci C, D’Alessandro P, Cantisani TA et al (1993) Epilepsy with bilateral occipital calcifications: Sturge-Weber variant or a different encephalopathy? Epilepsia 34:528-539

80. Fois A, Balestri P, Vascotto M et al (1993) Progressive cerebral calcifications, epilepsy, and celiac disease. Brain Dev 15:79-82

81. Gobbi G, Bouquet F, Greco L et al (1992) Coeliac disease, epilepsy, and cerebral calcifications. The Italian Working Group on Coeliac Disease and Epilepsy. Lancet 340:439-443

82. Crosato F, Senter S (1992) Cerebral occipital calcifications in celiac disease. Neuropediatrics 23:214-217

83. Ambrosetto G, Antonini L, Tassinari CA (1992) Occipital lobe seizures related to clinically asymptomatic celiac disease in adulthood. Epilepsia 33:476-481

84. Della Cella G, Beluschi C, Cipollina F (1991) Intracranial calcifications-seizures-celiac disease: a case presentation. Pediatr Med Chir 13:427-430

85. Ventura A, Bouquet F, Sartorelli C et al (1991) Coeliac disease, folic acid deficiency and epilepsy with cerebral calcifications. Acta Paediatr Scand 80:559-562
86. Hanly JG, Stassen W, Whelton M et al (1982) Epilepsy and coeliac disease. J Neurol Neurosurg Psychiatry 45:729-730

87. Banerji NK, Hurwitz LJ (1971) Neurological manifestations in adult steatorrhoea (probable Gluten enteropathy). J Neurol Sci $14: 125-141$

88. Morris JS, Ajdukiewicz AB, Read AE (1970) Neurological disorders and adult coeliac disease. Gut 11:549-554

89. Fiest KM, Sauro KM, Wiebe $\mathrm{S}$ et al. Prevalence and incidence of epilepsy: A systematic review and meta-analysis of international studies Neurology. 2017; 88:296-303

90. Kotsopoulos IA, van Merode T, Kessels FG et al (2002) Systematic review and meta-analysis of incidence studies of epilepsy and unprovoked seizures. Epilepsia 43:1402-1409

91. Hadjivassiliou M, Aeschlimann P, Strigun A et al (2008) Autoantibodies in gluten ataxia recognize a novel neuronal transglutaminase. Ann Neurol 64:332-343 